\title{
Selective Combining for Hybrid Cooperative Networks
}

\author{
Qiang Huo, Tianxi Liu, Shaohui Sun, Lingyang Song, and Bingli Jiao
}

\begin{abstract}
In this study, we consider the selective combining in hybrid cooperative networks (SCHCNs scheme) with one source node, one destination node and $N$ relay nodes. In the SCHCN scheme, each relay first adaptively chooses between amplify-and-forward protocol and decode-and-forward protocol on a per frame basis by examining the error-detecting code result, and $N_{c}\left(1 \leq N_{c} \leq N\right)$ relays will be selected to forward their received signals to the destination. We first develop a signal-to-noise ratio (SNR) threshold-based frame error rate (FER) approximation model. Then, the theoretical FER expressions for the SCHCN scheme are derived by utilizing the proposed SNR threshold-based FER approximation model. The analytical FER expressions are validated through simulation results.
\end{abstract}

\section{Index Terms}

Cooperative networks, frame error rate, hybrid forwarding scheme, relay selection, selective combining.

Published in IET Communications. Manuscript received May 5, 2013; revised August 26, 2013; accepted September 23 , 2013.

Q. Huo, L. Song, and B. Jiao are with the State Key Laboratory of Advanced Optical Communication Systems and Networks, and the Wireless Communications and Signal Processing Research Center, School of Electronics Engineering and Computer Science, Peking University, Beijing 100871, China (e-mail: \{qiang.huo, lingyang.song, jiaobl\}@pku.edu.cn).

T. Liu is with Datang Telecom Technology \& Industry Holdings, Beijing 100871, China (e-mail: Email: liutx@ datanggroup.cn).

S. Sun is with Peking University, Beijing 100871, China (e-mail: sunshaohui@ hotmail.com).

This work was partially supported by the National 973 project under grant number 2013CB336700, National Nature Science Foundation of China under grant number 61222104 and 61061130561, the Ph.D. Programs Foundation of Ministry of Education of China under grant number 20110001110102, and the Opening Project of Key Laboratory of Cognitive Radio and Information Processing (Guilin University of Electronic Technology).

Digital Object Identifier 10.1049/iet-com.2013.0323 


\section{INTRODUCTION}

Cooperative communication has been widely used to improve system robustness and capacity by allowing nodes to cooperate in their transmissions to form a virtual antenna array [1] $-[5]$. In [5], several relay protocols have been studied for the wireless cooperative networks, and amplify-and-forward (AF) and decode-and-forward (DF) are recognized as two basic cooperative transmission protocols. While using the AF protocol, the relay node amplifies the received signal sent by the source, and then retransmits it to the destination without decoding. In contrast, the DF protocol requires the relay node to decode the received signal and then forward the re-encoded signal to the destination. However, the performance of the AF is mainly limited by the noise amplification phenomenon at the relay nodes and the DF may cause serious error propagation problem when the relay fails to decode the received signal correctly [6]-[9].

Motivated by the above-described disadvantages of the AF and DF protocols, various improved cooperative transmission protocols have been proposed, such as signal-to-noise ratio (SNR) threshold-based selective digital relaying schemes [6], [10]-[12], cooperative maximum ratio combining (MRC)-based DF [13], [14] and smart relaying scheme [15], [16], log-likelihood-ratio (LLR) threshold-based selective DF [17], decode-amplify-forward (DAF) [18], [19]. A hybrid forward (HF) protocol was proposed in [8], [9], [20]-[23], which provides superior performance than both the AF and the DF protocols and thus has received lots of attention recently. In [8], [9], the author studied the symbol error rate (SER) of the HF protocol by adaptively choosing the AF and the DF protocols on a per symbol basis, which is, however, hard to realise in practice. This work focuses on a more practical HF protocol on a per frame basis.

In conventional cooperative wireless networks, all $N$ relays will forward the signals received from the source to the destination through $N$ orthogonal channels in the relaying phase [24]-[28]. Then the destination combines all the signals received from the source and the relays using MRC technique. In [27], [28], the authors show that the all participate AF (AP-AF) scheme can achieve the full diversity order of $N+1$. However, MRC, which combines the signals received from all the links in order to have the best possible combining gain, will certainly result in extremely complicated hardware implementation required for phase coherence and amplitude estimation on each link [29], [30]. Furthermore, note that the links with low SNRs may possibly lead to inefficient estimation on both phase and amplitude; hence MRC is sensitive to the channel estimation errors [31]. To simplify the hardware implementation and make the communication systems more robust towards channel estimation errors while still retaining good system performance, Kong et al. [32] first proposed the selection combining (SC) scheme at the receiver, which combines $N_{c}$ links with the largest instantaneous SNRs out of $N$ links, for a rake receiver 
operating over a multipath fading channel. As a result, the SC scheme has a fixed and low processing complexity since it only combines $N_{c}$ links, instead of all the $N$ links in the network [30]. In addition, in the SC scheme, the weakest SNR paths are excluded from the combining process to make the system more robust towards channel estimation errors.

Recently, the SC scheme was extended to the cooperative communication networks by selecting proper number of relay nodes to forward the signals to the destination for the source during the transmission phase. The work in [33] and [34] studies the SER performances of the SC with the AF and the DF protocols on a per symbol basis. Note that the conventional cooperative scheme [24]-[28], in which all $N$ relays will forward the signals to the destination, can be viewed as a special case of the SC scheme when $N_{c}=N$, and the conventional relay selection (RS) scheme [21], [22], [35], [36], in which only the relay that has the highest SNRs at the destination is chosen as the active relay, is also a special case of the SC scheme when $N_{c}=1$. Obviously, the SC scheme is able to describe the cooperative networks in a unified and convenient manner, and provides insight into the system designs. However, to the best of our knowledge, no results for the HF scheme with the SC have been reported in the literature so far. Furthermore, most earlier works have been focusing on SER performance analysis and limited works for the analytical frame error rate (FER) performance of the HF scheme, which is more frequently used in the evaluation of the system performance, have been presented. These motivated our work.

In this paper, we consider a two-hop network with one source node, one destination node and $N$ relay nodes, and with links experiencing independent block Rayleigh fading. The main contributions of the present work can be briefly summarized as follows:

1) We propose and study the selective combining in hybrid cooperative networks (SCHCN scheme) on a per frame basis. In the proposed SCHCNs scheme, each relay adaptively chooses, frame by frame, between the AF and DF protocols by examining the error-detecting code results. Specifically, we use cyclic redundancy check (CRC) codes for the AF and DF adaptation in this work. The relays, which decode correctly, will utilize the DF protocol, and the rest will operate in the AF mode. Meanwhile, $N_{c}$ relays that have the highest effective SNRs at the destination are chosen out of $N$ relays, and then retransmit their received signals to the destination.

2) We develop an SNR threshold-based FER approximation model and then establish the relationship between the outage probability and the FER by using a novel analytical SNR threshold.

3) Using the proposed FER approximation model, a closed-form FER expression as well as a high SNR asymptotic FER expression for the SCHCN scheme are derived. The derived FER expressions clearly indicate that the SCHCN scheme can achieve full diversity order. Analytical results are 
verified through simulations. Simulation results show that the best RS scheme outperforms the multiple RS scheme under a total transmit power constraint 1 , whereas the multiple RS scheme provides better performance than the best RS scheme under an individual transmit power constraint 2 .

The rest of the paper is organized as follows: we describe the system model and the SCHCN scheme in Section II In Section III, we develop an SNR threshold-based FER approximation model and derive an improved SNR threshold. In Section IV, we calculate the closed-form average FER and the simplified asymptotic FER expressions for the SCHCN scheme by using the FER approximation model. In Section V] we present some simulation results. In Section VI, we draw the main conclusions.

Notation: For a random variable $(\mathrm{RV}) X, \operatorname{Pr}(\cdot)$ represents its probability, $f_{X}(\cdot)$ represents its probability density function (PDF) and $F_{X}(\cdot)$ denotes its cumulative density function $(\mathrm{CDF})$. Let $\mathcal{L}_{X}(s)$ and $\hat{\mathcal{L}}_{X}(s)$ stand for the Laplace transform of the PDF and CDF of $X$, respectively. $X \sim \mathcal{C N}(0, \Omega)$ denotes a circular symmetric complex Gaussian variable with a zero mean and variance $\Omega$. $Q(x)$ represents the $Q$-function, that is, $Q(x)=\frac{1}{\sqrt{2 \pi}} \int_{x}^{\infty} e^{-t^{2} / 2} \mathrm{~d} t$.

\section{System Model AND THE SCHCN SCHEME}

\section{A. System Model}

We consider a general two-hop wireless relay network with $N+2$ terminals consisting of one source node, $N$ relay nodes and one destination node, as shown in Fig. 1. The channels of all links are assumed to be quasi-static Rayleigh fading, i.e., the channel is fixed within one frame and changes independently from one frame to another. Let $h_{0}, h_{1, i}$ and $h_{2, i}$ represent the channel coefficients of the source-destination, source-relay $i$ and relay $i$-destination channels, respectively. Let $h_{0} \sim \mathcal{C N}\left(0, \Omega_{0}\right), h_{1 i} \sim \mathcal{C N}\left(0, \Omega_{1 i}\right)$ and $h_{2 i} \sim \mathcal{C N}\left(0, \Omega_{2 i}\right)$. Let $n_{0}, n_{1 i}$ and $n_{2 i}$ represent the corresponding additive white Gaussian noises (AWGNs). It is assumed that $n_{0} \sim \mathcal{C N}\left(0, N_{0}\right), n_{1 i} \sim \mathcal{C N}\left(0, N_{0}\right)$ and $n_{2 i} \sim \mathcal{C N}\left(0, N_{0}\right)$. Finally, let $\mathcal{E}_{s}$ and $\mathcal{E}_{r, i}$ denote the average transmit power at the source and the $i$ th relay, respectively.

\section{B. Hybrid Forward Scheme}

It is assumed that each terminal in the network is in the half-duplex mode with single antenna. Signals are transmitted over orthogonal channels, either through time or frequency division, in order to prevent

\footnotetext{
${ }^{1}$ In some scenarios, such as sensor networks, energy efficiency emerges as a critical issue, and we can use the total transmit power constraint to portray the actual limitation.

${ }^{2}$ Here, we also investigate the case with individual transmit power constraint because in some relay scenarios, for example, fixed relay scenario [37], the battery problem is not so sensitive as the mobile user relay scenario.
} 
interference. Thus, one frame transmission in the relaying scheme consists of two separate phases, i.e., the broadcasting (1st) phase and the relaying (2nd) phase.

1) The Broadcasting Phase: The source broadcasts a signal to both the $N$ relays and the destination in the broadcasting phase. The received signals at the destination and the $i$ th relay, at time $t$, denoted by $y_{0}(t)$ and $y_{1 i}(t)$, can be expressed as

$$
y_{0}(t)=\sqrt{\mathcal{E}_{s}} h_{0} s(t)+n_{0}(t),
$$

and

$$
y_{1 i}(t)=\sqrt{\mathcal{E}_{s}} h_{1 i} s(t)+n_{1 i}(t),
$$

respectively, where $s(t)$ is the signal transmitted at the source. The corresponding instantaneous SNRs are given as $\gamma_{0}=\frac{\mathcal{E}_{s}}{N_{0}}\left|h_{0}\right|^{2}=\tilde{\gamma}_{0}\left|h_{0}\right|^{2}$ and $\gamma_{1 i}=\frac{\mathcal{E}_{s}}{N_{0}}\left|h_{1 i}\right|^{2}=\tilde{\gamma}_{0}\left|h_{1 i}\right|^{2}$, respectively, where $\tilde{\gamma}_{0}=\frac{\mathcal{E}_{s}}{N_{0}}$.

2) The Relaying Phase: During the relaying phase, each relay adaptively chooses, frame by frame, between the $\mathrm{AF}$ and $\mathrm{DF}$ protocols by examining the error-detecting code result. We use CRC codes for the $\mathrm{AF}$ and $\mathrm{DF}$ adaptation in this paper. The relays which decode correctly, i.e., the CRC checking result is correct, are included in the DF group, denoted by $\mathcal{G}_{D F}$, and the rest are included in the AF group, denoted by $\mathcal{G}_{A F}$.

The corresponding received signal at the destination, denoted by $y_{2 i}(t)$ can be written as

$$
y_{2 i}(t)=\sqrt{\mathcal{E}_{r, i}} h_{2 i} x_{i}(t)+n_{2 i}(t)
$$

where $x_{i}(t)$ represents the signal transmitted from the $i$ th relay. Note that the transmit signal $x_{i}(t)$ satisfies the following power constraint, $\mathbb{E}\left(\left|x_{i}(t)\right|^{2}\right) \leq 1$. Hence, the corresponding transmit signal $x_{i}(t)$ can be expressed as [5]

$$
x_{i}(t)= \begin{cases}\frac{y_{1 i}(t)}{\sqrt{\mathcal{E}_{s}\left|h_{1 i}\right|^{2}+N_{0}}} & \text { if } i \in \mathcal{G}_{A F}, \\ s(t) & \text { if } i \in \mathcal{G}_{D F} .\end{cases}
$$

Therefore the instantaneous SNR of the link through the $i$ th relay node, denoted by $\gamma_{i}$, is given as [38]

$$
\gamma_{i}= \begin{cases}\frac{\gamma_{1 i} \gamma_{2 i}}{\gamma_{1 i}+\gamma_{2 i}+1} & \text { if } i \in \mathcal{G}_{A F}, \\ \gamma_{2 i} & \text { if } i \in \mathcal{G}_{D F},\end{cases}
$$

where $\gamma_{2 i}$ is the instantaneous SNR of the link between the $i$ th relay and the destination, which is given by $\gamma_{2 i}=\frac{\mathcal{E}_{r, i}}{N_{0}}\left|h_{2 i}\right|^{2}=\tilde{\gamma}_{2 i}\left|h_{2 i}\right|^{2}$ and $\tilde{\gamma}_{2 i}=\frac{\mathcal{E}_{r, i}}{N_{0}}$. 


\section{SCHCN Scheme}

On receiving the signal sent from the source, each relay needs to send one bit indicator to inform the destination that it uses the AF or DF protocols. In the SCHCN scheme, destination then calculates the overall received SNR from each relay accordingly and selects the $N_{c}$ relays with the largest effective SNRs. After finding the optimum $N_{c}$ relays, through a feedback channel, the destination will inform which relays are selected for transmission during the relaying phrase and other unselected relays will be in an idle state. Let $\gamma_{(1)} \geq \gamma_{(2)} \geq \cdots \geq \gamma_{(N)}$ represent the decreasing ordered SNRs of the $\left\{\gamma_{n}\right\}_{n=1}^{N}$. Then, the instantaneous SNR of the MRC output at the destination is given by 3 [39]

$$
\gamma_{S C H C N}=\gamma_{0}+\sum_{n=1}^{N_{c}} \gamma_{(n)}=\gamma_{0}+\gamma_{\text {coop }}
$$

where $\gamma_{\text {coop }}=\sum_{n=1}^{N_{c}} \gamma_{(n)}$.

It is assumed that all channel state information (CSI) needed for decoding is available at the relay nodes and the destination node [5], [14], [16]. This can be easily achieved in practice. For example, the relay acquires the CSI of the source-relay channel via the pilot symbol sent from the source and then transmits it to the destination via a feedback channel. Similarly, the destination can acquire the CSI of the relay-destination via the pilot symbol sent from the relay. For simplicity, let $\lambda_{0}=\frac{1}{\tilde{\gamma}_{0} \Omega_{0}}, \lambda_{1 i}=\frac{1}{\tilde{\gamma}_{0} \Omega_{1 i}}$, and $\lambda_{2 i}=\frac{1}{\tilde{\gamma}_{2 i} \Omega_{2 i}}$. As $\gamma_{0}, \gamma_{1 i}$ and $\gamma_{2 i}$ are exponentially distributed, their PDFs can be expressed as

$$
f_{\gamma_{k}}(\gamma)=\lambda_{k} e^{-\lambda_{k} \gamma}, k \in\{0,1 i, 2 i\}
$$

It is also assumed that $\left\{\gamma_{1 i}\right\}_{i=1}^{N}\left(\left\{\gamma_{2 i}\right\}_{i=1}^{N}\right)$ are independent and identically distributed (i.i.d.) RVs and let $\lambda_{1 i}=\lambda_{s r}$ and $\lambda_{2 i}=\lambda_{r d}$ for analysis tractability in this work.

\section{SNR ThreshOlD-BASED FER APPROXIMATION MODEL}

In this section, an SNR threshold-based FER approximation model is proposed for diversity systems. As we know, cooperative systems can be viewed as a special kind of general diversity systems [5].

\footnotetext{
${ }^{3}$ It it noteworthy that, during the pilot phase, the destination has to estimate $N$ channels from the relay nodes and one channel from the source, and then selects the $N_{c}$ best relay nodes. Thus the complexity of the pilot phase, is mainly dominated by the $N+1$ channel estimation process. The exact complexity is dependent on the channel estimation approach, for example, when using zero-forcing (ZF) to estimate the channel, one needs $N+1$ floating-point operations. Furthermore, during the data transmission phase, the destination has to combine $N_{c}$ links from the relay nodes as well as the direct link from the source by using the MRC technique. Thus, the complexity of the data combining process requires $N_{c}+1$ multiplies and $N_{c}$ additions, i.e., $2 N_{c}+1$ floating-point operations.
} 
Therefore, we can apply the results obtained in this section to the FER analysis of the SCHCN scheme presented in next section.

In the following, the SNR threshold-based FER approximation model is described in Subsection $\amalg I-A$. some existing related works are presented in Subsection III-B and then an improved criterion and the corresponding SNR threshold are presented in Subsection III-C Finally, a multiple-input multipleoutput (MIMO) system using the proposed SNR threshold-based FER approximation model is presented as a simple example in Subsection $\amalg$

\section{A. SNR threshold-based FER approximation model}

Note that the average FER, represented by $\bar{P}_{f}$, can be calculated by integrating the instantaneous FER in AWGN channel, denoted by $P_{f}^{G}(\gamma)$, over the fading distribution [40]

$$
\bar{P}_{f}(\bar{\gamma})=\int_{0}^{\infty} P_{f}^{G}(\gamma) f_{\gamma}(\gamma, \bar{\gamma}) \mathrm{d} \gamma
$$

where $\gamma$ and $\bar{\gamma}$ represent the instantaneous and average SNR, respectively, and $f_{\gamma}(\cdot)$ represents the PDF of $\gamma$.

Eq. (8) is an exact expression to calculate $\bar{P}_{f}$; however, its closed-form expression is still difficult to be derived. The SNR threshold-based FER approximation model is an accurate and simple approach to evaluate FER. Assume that the instantaneous FER is 1 when the instantaneous SNR $\gamma$ is below a given SNR threshold $\gamma_{t}$, otherwise it is 0 [41]-[43], i.e.,

$$
P_{f}^{G}\left(\gamma \mid \gamma \leq \gamma_{t}\right) \approx 1 \text { and } P_{f}^{G}\left(\gamma \mid \gamma>\gamma_{t}\right) \approx 0
$$

Then, the average FER is given as [41]-[43]

$$
\begin{aligned}
\bar{P}_{f}(\bar{\gamma}) & =\int_{0}^{\gamma_{t}} P_{f}^{G}(\gamma) f_{\gamma}(\gamma, \bar{\gamma}) \mathrm{d} \gamma+\int_{\gamma_{t}}^{\infty} P_{f}^{G}(\gamma) f_{\gamma}(\gamma, \bar{\gamma}) \mathrm{d} \gamma \\
& \approx \int_{0}^{\gamma_{t}} f_{\gamma}(\gamma, \bar{\gamma}) \mathrm{d} \gamma=F_{\gamma}\left(\gamma_{t}, \bar{\gamma}\right)
\end{aligned}
$$

where $F_{\gamma}(\cdot)$ represents the $\mathrm{CDF}$ of $\gamma$.

According to the SNR threshold-based FER approximation model, the average FER is approximately calculated as an outage probability. Hence the accuracy of the FER model is principally determined by the value of the given SNR threshold.

\section{B. Related Works}

In [41], the SNR threshold-based FER approximation model is applied to the iteratively decoded systems with turbo codes. It is shown that the optimal SNR threshold coincides with the convergence 
threshold of the iterative turbo decoder. Furthermore, the SNR threshold-based FER model is extended to non-iterative coded and uncoded systems in [42], [43]. The minimum absolute error sum criterion is adopted in [42], [43] to minimize the sum of absolute error

$$
\gamma_{t}=\arg \min _{\gamma}\left\{\int_{0}^{\infty}\left|\bar{P}_{f}(\bar{\gamma})-F_{\gamma}(\gamma, \bar{\gamma})\right| \mathrm{d} \bar{\gamma}\right\}
$$

where $\bar{\gamma}$ denotes the average SNR, and the SNR threshold is given as

$$
\gamma_{t}=\left(\int_{0}^{\infty} \frac{1-P_{f}^{G}(\gamma)}{\gamma^{2}} \mathrm{~d} \gamma\right)^{-1} .
$$

Since the minimum absolute error sum criterion does not consider the fact that the FER decreases more quickly at high SNR region in high diversity order systems, it needs to be improved for general diversity order systems.

\section{Improved SNR Threshold}

A new SNR threshold-based FER approximation model is developed for the diversity systems based on two important aspects.

Firstly, a minimum relative error sum criterion, which taking into account the fact that the FER decreases quickly when SNR increases and minimizing the sum of relative error, is used instead of the minimum absolute error sum criterion in Eq. (11) as

$$
\gamma_{t}=\arg \min _{\gamma}\left\{\int_{0}^{\infty}\left|\frac{\bar{P}_{f}(\bar{\gamma})-F_{\gamma}(\gamma, \bar{\gamma})}{\bar{P}_{f}(\bar{\gamma})}\right| \mathrm{d} \bar{\gamma}\right\} .
$$

In the Appendix $\mathrm{A}$, we will show that the minimum relative error sum criterion will lead to a minimum relative error criterion at high $S N R$ as

$$
\gamma_{t}=\arg \lim _{\gamma, \bar{\gamma} \rightarrow \infty}\left\{\bar{P}_{f}(\bar{\gamma})-F_{\gamma}(\gamma, \bar{\gamma})=0\right\} .
$$

Secondly, the calculation of SNR threshold should take into account the factor of the diversity order. We note that in a Rayleigh fading channel, the $\operatorname{CDF}$ of SNR is given by $F_{\gamma}(\gamma, \bar{\gamma})=1-e^{-\gamma / \bar{\gamma}}$, then we have $\lim _{\bar{\gamma} \rightarrow \infty} F_{\gamma}(\gamma, \bar{\gamma})=\bar{\gamma}^{-1} \gamma$. Similarly, in a Nakagami- $m$ fading channel, the CDF of SNR is $F_{\gamma}(\gamma, \bar{\gamma})=\frac{\Gamma(m, m \gamma / \bar{\gamma})}{\Gamma(m)}$, where $\Gamma(m)$ denotes the Gamma function, and $\Gamma(m, x)$ denotes the lower part incomplete Gamma function given by $\Gamma(m, x)=\int_{0}^{x} t^{m-1} e^{-t} \mathrm{~d} t$. At high SNR, we have $\lim _{\bar{\gamma} \rightarrow \infty} F_{\gamma}(\gamma, \bar{\gamma})=\Gamma(m)^{-1} \int_{0}^{m \gamma / \bar{\gamma}} t^{m-1} \mathrm{~d} t=\frac{m^{m-1}}{\Gamma(m)(\bar{\gamma})^{m}} \gamma^{m}$. Generally speaking, the CDF of a system with a diversity order of $d$ at high SNR can be approximated as [43]-[45]:

$$
\lim _{\bar{\gamma} \rightarrow \infty} F_{\gamma}(\gamma, \bar{\gamma}) \rightarrow G(\bar{\gamma}) \gamma^{d}
$$


where $G(\bar{\gamma})$ represents a constant related to $\bar{\gamma}$.

Combining Eq. (8), Eq. (14) and Eq. (15), we derive the closed-form improved SNR threshold as

$$
\begin{aligned}
\gamma_{t, d} & =\arg \lim _{\gamma_{t}, \bar{\gamma} \rightarrow \infty}\left\{\int_{0}^{\infty} f_{\gamma}(\gamma, \bar{\gamma}) P_{f}^{G}(\gamma) \mathrm{d} \gamma-G(\bar{\gamma}) \gamma_{t}^{d}=0\right\} \\
& =\arg \lim _{\gamma_{t}, \bar{\gamma} \rightarrow \infty}\left\{\int_{0}^{\infty} G(\bar{\gamma}) d \gamma^{d-1} P_{f}^{G}(\gamma) \mathrm{d} \gamma-G(\bar{\gamma}) \gamma_{t}^{d}=0\right\} \\
& =\arg \lim _{\gamma_{t}, \bar{\gamma} \rightarrow \infty}\left\{\gamma_{t}^{d}=d \int_{0}^{\infty} \gamma^{d-1} P_{f}^{G}(\gamma) \mathrm{d} \gamma\right\} \\
& =\left(d \int_{0}^{\infty} \gamma^{d-1} P_{f}^{G}(\gamma) \mathrm{d} \gamma\right)^{1 / d} .
\end{aligned}
$$

Note that $P_{f}^{G}(\gamma)$ can be calculated in closed-form for uncoded systems. For example, for linear modulation, $P_{f}^{G}(\gamma)$ is given as [40], [42]

$$
P_{f}^{G}(\gamma)=1-(1-Q(\sqrt{c \gamma}))^{L}
$$

where $c$ represents modulation constant ( $c=2$ for binary-phase-shift-keying (BPSK)), $Q(\cdot)$ represents the $Q$ function, and $L$ denotes the frame length.

Substituting Eq. (17) into Eq. (16), the SNR threshold $\gamma_{t, d}$ for uncoded systems is given as:

$$
\gamma_{t, d}=\left(d \int_{0}^{\infty} \gamma^{d-1}\left(1-(1-Q(\sqrt{c \gamma}))^{L}\right) \mathrm{d} \gamma\right)^{1 / d} .
$$

In Table I] we present the SNR thresholds by using Eq. (18) for the systems with different diversity orders when $c=2$.

We note that when substituting $c=2$ (BPSK), $L=1$ and $d=1$ into Eq. (18) and with the help of [46. Eq. (6.313.2)], we have $\gamma_{t, 1}=\frac{1}{4}$ and $\bar{P}_{f}=1-e^{-\frac{1}{4 \bar{\gamma}}} \approx \frac{1}{4 \bar{\gamma}}$, which is equivalent to the average BER of BPSK over Rayleigh fading channel at high SNR: $\bar{P}_{b}=\frac{1}{2}\left(1-\sqrt{\frac{\bar{\gamma}}{1+\bar{\gamma}}}\right) \approx \frac{1}{4 \bar{\gamma}}[40]$.

\section{Simple Example of MIMO Systems}

Let us use a MIMO system as a simple example to illustrate the usage of the developed SNR thresholdbased FER model. Assume that the MIMO system has $N_{T}$ inputs and $N_{R}$ outputs, and the transmitter uses space-time block coding (STBC) [47]. The receiver use MRC technique to combine the $N=N_{T} N_{R}$ independent fading paths, and the PDF and CDF of the instantaneous SNR, represented by $\gamma$, at the output of the combiner are given by [40], [43]:

$$
f_{\gamma}(\gamma, \bar{\gamma})=\frac{\gamma^{N-1} e^{-\gamma /\left(\bar{\gamma} / N_{T}\right)}}{\left(\bar{\gamma} / N_{T}\right)^{N}(N-1) !}
$$


and

$$
F_{\gamma}(\gamma, \bar{\gamma})=1-e^{-\gamma N_{T} / \bar{\gamma}} \sum_{k=0}^{N-1} \frac{\left(\gamma N_{T} / \bar{\gamma}\right)^{k}}{k !},
$$

respectively, where $\bar{\gamma}$ represents the average SNR per receive antenna.

It should be mentioned here that the approximation in Eq. (15) still holds for the MIMO system.

Proof: With the fact that $\lim _{\bar{\gamma} \rightarrow \infty} e^{-\gamma N_{T} / \bar{\gamma}}=1$, we have

$$
\begin{aligned}
\lim _{\bar{\gamma} \rightarrow \infty} F_{\gamma}(\gamma, \bar{\gamma}) & =\lim _{\bar{\gamma} \rightarrow \infty} \int_{0}^{\gamma} f_{\gamma}(t, \bar{\gamma}) \mathrm{d} t \\
& =\int_{0}^{\gamma} \frac{t^{N-1}}{\left(\bar{\gamma} / N_{T}\right)^{N}(N-1) !} \mathrm{d} t \\
& =\frac{1}{\left(\bar{\gamma} / N_{T}\right)^{N} N !} \gamma^{N} \\
& =G(\bar{\gamma}) \gamma^{N},
\end{aligned}
$$

where $G(\bar{\gamma})=\frac{1}{\left(\bar{\gamma} / N_{T}\right)^{N} N !}$.

In the following, with the derived SNR threshold $\gamma_{t, N}$ in Subsection ЩI-C, the FER of the MIMO system in quasi-static fading channel is approximately calculated as

$$
\begin{aligned}
\bar{P}_{f}(\bar{\gamma}) & \approx F_{\gamma}\left(\gamma_{t, N}, \bar{\gamma}\right) \\
& =1-e^{-\gamma_{t, N} N_{T} / \bar{\gamma}} \sum_{k=0}^{N-1} \frac{\left(\gamma_{t, N} N_{T} / \bar{\gamma}\right)^{k}}{k !} .
\end{aligned}
$$

In the simulation section, we compare the proposed SNR threshold obtained in Eq. (16) and the optimal SNR threshold obtained in Eq. $[23]^{4}$ by using numerical method for the MIMO system. Simulation results show that the proposed SNR threshold converges well with the optimal SNR threshold especially at medium and high SNRs, which justifies the proposed FER approximation model.

$$
\gamma_{t, N}^{o p t}=F_{\gamma}^{-1}\left(\bar{P}_{f}(\bar{\gamma}), \bar{\gamma}\right)
$$

So far, an improved SNR threshold-based FER approximation model is developed for diversity systems. In this developed FER model, the average FER is approximately calculated as an outage probability as Eq. (10), the improved SNR threshold can be calculated using Eq. (16) for general systems and can be calculated using Eq. (18) for uncoded systems. In the next section, we will apply this model to the FER analysis of the SCHCN scheme for cooperative systems. We should emphasize that our analysis in this work can be easily extended to the coded systems. However, the corresponding details for coded systems are omitted here because of limited space and please refer to [38], [52] for details.

\footnotetext{
${ }^{4} F_{\gamma}^{-1}(\cdot, \bar{\gamma})$ represents the inverse function of $F_{\gamma}(\gamma, \bar{\gamma})$ with respect to $\gamma$.
} 


\section{FER ANALYSIS}

We first apply the SNR threshold-based FER approximation model to calculate the closed-form analytical FER of the SCHCN scheme in Subsection IV-A. We then present the asymptotic FER expression for the SCHCN scheme in Subsection IV-B.

\section{A. Closed-form analytical FER}

The following properties for the SCHCN scheme are first established before performing FER calculation.

Property 1: The CDF of the end-to-end SNR of the indirect link $S \rightarrow R_{i} \rightarrow D$ for the SCHCN scheme can be expressed as

$$
F_{\gamma_{i}}(\gamma) \approx \begin{cases}1-e^{-\left(\lambda_{s r}+\lambda_{r d}\right) \gamma}, & \text { if } \gamma<\gamma_{t, 1}, \\ 1-e^{-\lambda_{s r} \gamma_{t, 1}} e^{-\lambda_{r d} \gamma}, & \text { if } \gamma \geq \gamma_{t, 1} .\end{cases}
$$

For simplicity, Eq. (24) can be further approximated by an exponential function to extend the range of $\gamma$ from $\gamma_{t, 1}$ to $\gamma_{t, d}$

$$
F_{\gamma_{i}}(\gamma) \approx 1-e^{-\lambda_{e q} \gamma}, 0 \leq \gamma \leq \gamma_{t, d}
$$

where the lower bound and upper bound of $\lambda_{e q}$ are $\lambda_{s r} \gamma_{t, 1} / \gamma_{t, d}+\lambda_{r d}$ and $\lambda_{s r}+\lambda_{r d}$, respectively. From Table \, we note that $\gamma_{t, 1} / \gamma_{t, d} \approx 1$ which implies that either the lower bound or upper bound can be used to approximate Eq. (24) and the approximation is tight.

Proof: Please see Appendix B]

Property 2: Let $\left\{\gamma_{i}\right\}_{i=1}^{N}$ be i.i.d. exponential RVs, represented by $\gamma_{i} \sim \mathcal{E}\left(\lambda_{e q}\right)$. Let $\gamma_{(1)} \geq \gamma_{(2)} \geq \cdots \geq$ $\gamma_{(N)}$ denote the order statistics obtained by arranging the $\left\{\gamma_{n}\right\}_{n=1}^{N}$ in a decreasing order. According to Eq. (6), the CDF of $\gamma_{S C H C N}$ in closed-form expression is derived for the two cases of both $\lambda_{0} \neq \lambda_{e q}$ and $\lambda_{0}=\lambda_{e q}$ as follows

a) Case of $\lambda_{0} \neq \lambda_{e q}$ :

$$
\begin{aligned}
F_{\gamma_{S C H C N}}\left(\gamma, \lambda_{e q}\right)= & \sum_{i=1}^{N_{c}} \frac{\alpha_{i}}{\lambda_{e q}^{i}}\left[1-e^{-\lambda_{e q} \gamma} \sum_{m=0}^{i-1} \frac{\left(\lambda_{e q} \gamma\right)^{m}}{m !}\right] \\
& +\sum_{j=1}^{N-N_{c}} \frac{\beta_{j}}{\left(1+\frac{j}{N_{c}}\right) \lambda_{e q}}\left(1-e^{-\left(1+\frac{j}{N_{c}}\right) \lambda_{e q} \gamma}\right) \\
& +\frac{\beta_{0}}{\lambda_{0}}\left(1-e^{-\lambda_{0} \gamma}\right)
\end{aligned}
$$


where $5=\frac{N !}{N_{c} ! N_{c}^{N-N_{c}}} \lambda_{0} \lambda_{e q}^{N}, \theta_{0}=\frac{1}{\prod_{n=1}^{N-N_{c}}\left[\left(1+\frac{n}{N_{c}}\right) \lambda_{e q}-\lambda_{0}\right]}, \theta_{k}=\frac{1}{\left[-\left(1+\frac{k}{N_{c}}\right) \lambda_{e q}+\lambda_{0}\right] \prod_{n=1, n \neq k}^{N-N_{c}}\left[\left(\frac{n-k}{N_{c}}\right) \lambda_{e q}\right]}, \alpha_{i}=$ $\frac{c}{\left(N_{c}-i\right) !}\left[\frac{\theta_{0}(-1)^{N_{c}-i}\left(N_{c}-i\right) !}{\left(-\lambda_{e q}+\lambda_{0}\right)^{N_{c}-i+1}}+\sum_{k=1}^{N-N_{c}} \frac{\theta_{k}(-1)^{N_{c}-i}\left(N_{c}-i\right) !}{\left(\frac{k}{N_{c}} \lambda_{e q}\right)^{N_{c}-i+1}}\right], \beta_{0}=\frac{c \cdot \theta_{0}}{\left(\lambda_{e q}-\lambda_{0}\right)^{N_{c}}}$ and $\beta_{j}=\frac{c \cdot \theta_{j}}{\left(-\frac{j}{N_{c}} \lambda_{e q}\right)^{N_{c}}}$.

b) Case of $\lambda_{0}=\lambda_{e q}$ :

$$
\begin{aligned}
F_{\gamma_{S C H C N}}\left(\gamma, \lambda_{e q}\right)= & \sum_{i=1}^{N_{c}+1} \frac{\alpha_{i}^{*}}{\lambda_{e q}^{i}}\left[1-e^{-\lambda_{0} \gamma} \sum_{m=0}^{i-1} \frac{\left(\lambda_{0} \gamma\right)^{m}}{m !}\right] \\
& +\sum_{j=1}^{N-N_{c}} \frac{\beta_{j}^{*}}{\left(1+\frac{j}{N_{c}}\right) \lambda_{0}}\left(1-e^{-\left(1+\frac{j}{N_{c}}\right) \lambda_{0} \gamma}\right),
\end{aligned}
$$

where $6 c^{*}=\frac{N !}{N_{c} ! N_{c}^{N-N_{c}}} \lambda_{0}^{N+1}, \theta_{k}^{*}=\frac{1}{\prod_{n=1, n \neq k}^{N-N_{c}}\left[\left(\frac{n-k}{N_{c}}\right) \lambda_{0}\right]}, \alpha_{i}^{*}=\frac{c^{*}}{\left(N_{c}+1-i\right) !}\left[\sum_{k=1}^{N-N_{c}} \frac{\theta_{k}^{*}(-1)^{N_{c}+1-i}\left(N_{c}+1-i\right) !}{\left(\frac{k}{N_{c}} \lambda_{0}\right)^{N_{c}-i+2}}\right]$ and $\beta_{j}^{*}=\frac{c^{*} \cdot \theta_{j}^{*}}{\left(-\frac{j}{N_{c}} \lambda_{0}\right)^{N_{c}+1}}$.

Proof: Please see Appendix C]

According to the SNR threshold-based FER approximation model in Section III the average FER of the SCHCN scheme can be expressed as an outage probability with an appropriate SNR threshold. Thus, the average FER of the SCHCN scheme, denoted by $\bar{P}_{f}$, is given as

$$
\bar{P}_{f} \approx F_{\gamma_{S C H C N}}\left(\gamma_{t, d}, \lambda_{e q}\right)
$$

where $d$ denotes the diversity order of the cooperative system using the SCHCN scheme and the calculation methods of parameters $\gamma_{t, d}$ are presented in Section $\amalg$. The accuracy of the derived close-form FER expression, i.e., Eq. (28), is verified through simulation results in the next section.

\section{B. Asymptotic FER}

In this subsection, we derive the asymptotic FER for the SCHCN scheme at high SNR.

Property 3: The asymptotic CDF of $\gamma_{S C H C N}$ at high SNR can be written in closed-form as

$$
F_{\gamma_{S C H C N}}\left(\gamma, \lambda_{e q}\right) \approx \frac{\lambda_{0} \lambda_{e q}{ }^{N} \gamma^{N+1}}{(N+1) N_{c} ! N_{c}^{N-N_{c}}} .
$$

Proof: Please see Appendix D,

With the help of Property 3, we can obtain the asymptotic FER expression of the SCHCN scheme as

$$
\bar{P}_{f} \approx F_{\gamma_{S C H C N}}\left(\gamma_{t, d}, \lambda_{e q}\right) \approx \frac{\lambda_{0} \lambda_{e q}{ }^{N} \gamma_{t, d}^{N+1}}{(N+1) N_{c} ! N_{c}^{N-N_{c}}} .
$$

\footnotetext{
${ }^{5}$ Note that as a special case of $N_{c}=N$, the following parameters are reduced to $c=\lambda_{0} \lambda_{e q}^{N}, \theta_{0}=1, \theta_{k}=0, \alpha_{i}=$ $\frac{c}{(N-i) !}\left[\frac{\theta_{0}(-1)^{N-i}(N-i) !}{\left(-\lambda_{e q}+\lambda_{0}\right)^{N-i+1}}\right], \beta_{0}=\frac{c \cdot \theta_{0}}{\left(\lambda_{e q}-\lambda_{0}\right)^{N}}$, and $\beta_{j}=0$.

${ }^{6}$ It is noteworthy that as a special case of $N_{c}=N$, the following parameters are reduced to $c^{*}=\lambda_{0}^{N+1}, \theta_{k}^{*}=0, \alpha_{i}^{*}=$ $0(i \neq N+1), \alpha_{N+1}^{*}=c^{*}$, and $\beta_{j}^{*}=0$.
} 
From Eq. (30) it shows that full diversity order of $N+1$ can be achieved in the SCHCN scheme, and thus, $\gamma_{t, d}=\gamma_{t, N+1}$. We should emphasize that the asymptotic FER is more simple and intuitive compared with the FER expression in Eq. (28).

\section{Simulation Results}

In this section, the analytical and the simulated results are provided for the SCHCN scheme. Without specific mention, all simulations are performed by a BPSK modulation and a frame size of 100 symbols over block Rayleigh fading channels [53]-[55]. We consider the multiple relay nodes scenario, e.g., $N=1,2,3$. Specifically, $N_{c}=0$ corresponds to the direct transmit case, and thus, no relay node is selected. $N_{c}=1$ denotes the best RS, and all the relay nodes are participated for the transmission when $N_{c}=N$. Without specific mention, we assume that a fixed total energy per symbol constraint in the network and equal power division among cooperating nodes, i.e., $\left(\mathcal{E}_{s}=\mathcal{E}_{r, i}=\frac{\mathcal{E}}{N_{c}+1}\right)$, and $\lambda_{e q}=\lambda_{s r}+\lambda_{r d}$. We also take into account the relay's location as: 1). the symmetric case, where relays are placed halfway between the source and destination and 2). the asymmetric case, where relays are close to the source or destination. In Table I] we show the SNR thresholds derived in Section [II for the systems of different diversity order. The simulation scenarios in this section are shown in Table $\amalg$.

In Fig. 2] we compare our proposed FER model in Section [II] with the FER model in [42] for case 0. For case 0, the SNR thresholds are found to be $4.61 \mathrm{~dB}$ based on the FER model in [42], i.e., Eq. (12), and are found to be $5.10 \mathrm{~dB}, 5.36 \mathrm{~dB}$ and $5.89 \mathrm{~dB}$ for $N=1,2,4$, respectively, based on our proposed FER model, i.e., Eq. (16). Fig. 2 shows that our proposed model matches well with the simulated FER as the SNR increases while the gap between the FER model in [42] and the simulated FER cannot be ignored even at high SNR. We also note that, as the diversity order increases, our proposed model is still accurate; however the FER results based on the model in [42] become less accurate.

In Fig. 3, we present the optimal SNR threshold, i.e., Eq. (23), obtained by using the numerical method, when the FER well matches the outage probability. We also include the proposed SNR threshold, i.e., Eq. (16), and the SNR threshold of [42], i.e., Eq. (12). It can be observed that our proposed SNR threshold converges to the optimal SNR threshold at medium and high SNR regimes quickly, which validates our analysis in Section $\amalg$.

In Fig. 4 and Fig. 5, we show the closed-form FER, asymptotic FER and simulated FER results of the SCHCN scheme for symmetric case, i.e., case 1. From Fig. 4, we can see that the closed-form curves given by Eq. (28) match well with the simulated results and Fig. 5 shows that the asymptotic results given by Eq. (30) converge to the simulated results at high SNRs. These validate our derived analytical 
FERs in Section III] In addition, Fig. 6 indicates that the derived FER of the SCHCN scheme is also accurate for the case 2 (asymmetric case). Case 3 is also verified through simulation, but omitted here for brevity. Hence, our FER analysis is valid for both symmetric case and asymmetric case.

Fig. 4 shows that the best RS provides superior performance than the multiple RS under a total transmit power constraint, i.e., $\mathcal{E}_{s}=\mathcal{E}_{r, i}=\frac{\mathcal{E}}{N_{c}+1}$, whereas Fig. 5 shows that the multiple RS outperforms the best RS under an individual transmit power constraint i.e., $\mathcal{E}_{s}=\mathcal{E}_{r, i}=\frac{\mathcal{E}}{N+1}$. Moreover, from Fig. 5 we note that the cooperative system with $N_{c}=2$ has very slight quality deterioration compared to the cooperative system with $N_{c}=3$. However, the cooperative system with $N_{c}=2$ requires lower implementation complexity and is more robust towards channel estimation errors. Hence, the SCHCN scheme has more merits than the conventional cooperative scheme in the practical systems for both with and without total transmit power constraint cases.

In Fig. 7, we compare the SCHCN scheme with the non-cooperative system, i.e., $N_{c}=0$. It is obviously shown that the SCHCN scheme can provide superior performance than the non-cooperative system, especially at high SNRs. This is easy to understand as the cooperative system achieves a full diversity order of $N+1$ as long as $N_{c}>0$, whereas the non-cooperative system only has the diversity of 1. The simulation results of the SCHCN scheme with various frame lengths are shown in Fig. 8, From Fig. 8, it shows that as the frame length decreases, the FER decreases. This finding is consistent with $[48]$

\section{Vi. CONCLUSiONS}

In this paper, we have analyzed the average FER of the SCHCN scheme in cooperative wireless networks. Specifically, we considered a two-hop network with one source node, one destination node and multiple relay nodes. The closed-form average FER expression as well as the asymptotic FER expression at high SNRs have been derived for the SCHCN scheme. Theoretical analysis closely matches the simulated results. Simulations also indicate that the best RS can achieve better performance than the multiple RS under a total transmit power constraint, whereas the multiple RS outperforms the best RS under an individual transmit power constraint.

\footnotetext{
${ }^{7}$ We would refer the reader to [48] for more details on the choice of a proper frame length.
} 


\section{APPENDIX A}

PROOF OF EQ. (14)

We notice that the relative error at high SNR cannot be ignored because the integration in Eq. (13) is from 0 to $\infty$. Hence, we should have $\lim _{\bar{\gamma} \rightarrow \infty}\left|\frac{\bar{P}_{f}(\bar{\gamma})-F_{\gamma}\left(\gamma_{t}, \bar{\gamma}\right)}{\bar{P}_{f}(\bar{\gamma})}\right| \rightarrow 0$, which leads to Eq. (14).

Otherwise, suppose that existing a sufficiently big value $T(0<T<\infty)$ and a small enough value $\delta(0<\delta<\infty)$, the absolute relative error can be greater than $\delta$, i.e. $\left|\frac{\bar{P}_{f}(\bar{\gamma})-F_{\gamma}\left(\gamma_{t}, \bar{\gamma}\right)}{\bar{P}_{f}(\bar{\gamma})}\right|>\delta$, when $\bar{\gamma}>T$. Note that the sum of absolute relative error cannot be minimized in this situation as it will approach infinity: $\int_{0}^{\infty}\left|\frac{\bar{P}_{f}(\bar{\gamma})-F_{\gamma}\left(\gamma_{t}, \bar{\gamma}\right)}{P_{f}(\bar{\gamma})}\right| \mathrm{d} \bar{\gamma}>\int_{T}^{\infty} \delta \mathrm{d} \bar{\gamma}=\infty$. Thus, Eq. (14) is proved.

\section{APPENDIX B}

CDF of $\gamma_{i}$ IN The SCHCN scheme: The Proof of EQ. (24) AND EQ. (25)

Note that the CDF of $\gamma_{i}$ is given as

$$
\begin{aligned}
F_{\gamma_{i}}(\gamma)= & \operatorname{Pr}\left(\gamma_{i}<\gamma\right) \\
= & \operatorname{Pr}\left(i \in \mathcal{G}_{A F}\right) \operatorname{Pr}\left(\gamma_{i}<\gamma \mid i \in \mathcal{G}_{A F}\right) \\
& +\operatorname{Pr}\left(i \in \mathcal{G}_{D F}\right) \operatorname{Pr}\left(\gamma_{i}<\gamma \mid i \in \mathcal{G}_{D F}\right) .
\end{aligned}
$$

In the HF scheme, the relays which decode correctly are included in the $\mathcal{G}_{D F}$ and the rest are included in the $\mathcal{G}_{A F}$. According to our proposed FER approximation model, a frame error only occurs when SNR is below the SNR threshold $\gamma_{t}$. If $\gamma_{1 i} \geq \gamma_{t, 1}$ then $i \in \mathcal{G}_{D F}$, and if $\gamma_{1 i}<\gamma_{t, 1}$ then $i \in \mathcal{G}_{A F}$. Hence, we have $\operatorname{Pr}\left(i \in \mathcal{G}_{A F}\right)=1-e^{-\lambda_{s r} \gamma_{t, 1}}$ and $\operatorname{Pr}\left(i \in \mathcal{G}_{D F}\right)=e^{-\lambda_{s r} \gamma_{t, 1}}$, respectively.

If $i \in \mathcal{G}_{D F}, \gamma_{i}=\gamma_{2 i}$, then the conditional CDF of $\gamma_{i}$ given $i \in \mathcal{G}_{D F}$ can be expressed as

$$
\operatorname{Pr}\left(\gamma_{i}<\gamma \mid i \in \mathcal{G}_{D F}\right)=\operatorname{Pr}\left(\gamma_{2 i}<\gamma\right)=1-e^{-\lambda_{r d} \gamma}
$$

If $i \in \mathcal{G}_{A F}$, using the approximation $\frac{x y}{x+y+1} \approx \min \{x, y\}$ [25], then the corresponding conditional CDF of $\gamma_{i}$ given $i \in \mathcal{G}_{A F}$ can be expressed as [49]

$$
\begin{aligned}
& \operatorname{Pr}\left(\gamma_{i}<\gamma \mid i \in \mathcal{G}_{A F}\right) \\
\approx & \operatorname{Pr}\left(\min \left\{\left.\gamma_{1 i}\right|_{i \in \mathcal{G}_{A F}}, \gamma_{2 i}\right\}<\gamma\right) \\
= & 1-\left(1-\operatorname{Pr}\left(\gamma_{1 i}<\gamma \mid i \in \mathcal{G}_{A F}\right)\right)\left(1-\operatorname{Pr}\left(\gamma_{2 i}<\gamma\right)\right),
\end{aligned}
$$

where the conditional $\mathrm{CDF}$ of $\gamma_{1 i} \mid i \in \mathcal{G}_{A F}$ is given by

$$
\operatorname{Pr}\left(\gamma_{1 i}<\gamma \mid i \in \mathcal{G}_{A F}\right)= \begin{cases}\frac{1-e^{-\lambda_{s r} \gamma}}{1-e^{-\lambda_{s r} \gamma_{t}, 1}}, & \text { if } \gamma<\gamma_{t, 1} \\ 1 . & \text { if } \gamma \geq \gamma_{t, 1}\end{cases}
$$


Combining Eq. (33) and Eq. (34), the corresponding conditional CDF of $i \in \mathcal{G}_{A F}$ can be calculated as

$$
\operatorname{Pr}\left(\gamma_{i}<\gamma \mid i \in \mathcal{G}_{A F}\right) \approx \begin{cases}1-\frac{e^{-\lambda_{s r} \gamma}-e^{-\lambda_{s r} \gamma_{t, 1}}}{1-e^{-\lambda_{s r} \gamma_{t, 1}}} e^{-\lambda_{r d} \gamma}, & \text { if } \gamma<\gamma_{t, 1}, \\ 1, & \text { if } \gamma \geq \gamma_{t, 1} .\end{cases}
$$

Thus, after doing some substitutions in Eq. (31), it yields Eq. (24). When $\gamma_{t, 1} \leq \gamma \leq \gamma_{t, d}$, we use an exponential function to approximate Eq. (24). Let $1-e^{-\lambda_{s r} \gamma_{t, 1}} e^{-\lambda_{r d} \gamma}=1-e^{-\lambda_{e q} \gamma}$, that is, $\lambda_{e q}=\lambda_{s r} \gamma_{t, 1} / \gamma+\lambda_{r d}$. Hence, we have $\lambda_{s r} \gamma_{t, 1} / \gamma_{t, d}+\lambda_{r d} \leq \lambda_{e q} \leq \lambda_{s r}+\lambda_{r d}$. We note that this inequality still holds when considering the whole range from 0 to $\gamma_{t, d}$.

\section{APPENDIX C}

\section{PROOF OF PROPERTY 2}

According to Eq. (6), $\gamma_{S C H C N}=\gamma_{0}+\gamma_{c o o p}$, in the following, we first derive the Laplace transforms of the PDF of $\gamma_{\text {coop }}$ and $\gamma_{0}$, and then the PDF of $\gamma_{S C H C N}$ can be directly obtained by using the inverse Laplace transform.

As $\left\{\gamma_{i}\right\}_{i=1}^{N}$ are i.i.d. exponential RVs, with the help of [50, Eq. (9.321)], the Laplace transform of the PDF of $\gamma_{\text {coop }}$ can be expressed as

$$
\begin{aligned}
\mathcal{L}_{\gamma_{\text {coop }}}(s) & =\frac{1}{\left(1+\frac{s}{\lambda_{e q}}\right)^{N_{c}-1} \prod_{n=N_{c}}^{N}\left(1+\frac{s N_{c}}{\lambda_{e q} n}\right)} \\
& =\frac{\frac{N !}{N_{c} ! N_{c}^{N-N_{c}}} \lambda_{e q}^{N}}{\left(s+\lambda_{e q}\right)^{N_{c}} \prod_{n=1}^{N-N_{c}}\left[s+\left(1+\frac{n}{N_{c}}\right) \lambda_{e q}\right]},
\end{aligned}
$$

and with the help of [46, Eq. (17.13.7)], the Laplace transform of the PDF of $\gamma_{0}$ can be expressed as

$$
\mathcal{L}_{\gamma_{0}}(s)=\frac{\lambda_{0}}{s+\lambda_{0}}
$$

As $\gamma_{\text {coop }}$ and $\gamma_{0}$ are mutually independent, the Laplace transforms of the PDF of $\gamma_{S C H C N}$ is given as

$$
\begin{aligned}
\mathcal{L}_{\gamma_{S C H C N}}(s) & =\mathcal{L}_{\gamma_{\text {coop }}}(s) \cdot \mathcal{L}_{\gamma_{0}}(s) \\
& =\frac{\frac{N !}{N_{c} ! N_{c}^{N-N_{c}}} \lambda_{0} \lambda_{e q}^{N}}{\left(s+\lambda_{e q}\right)^{N_{c}}\left(s+\lambda_{0}\right) \prod_{n=1}^{N-N_{c}}\left[s+\left(1+\frac{n}{N_{c}}\right) \lambda_{e q}\right]} .
\end{aligned}
$$

Note that Eq. (38) can be rewritten for the two cases $\lambda_{0} \neq \lambda_{e q}$ and $\lambda_{0}=\lambda_{e q}$ as follows. 
1) Case of $\lambda_{0} \neq \lambda_{e q}$ : Applying the partial fraction expansion [51], Eq. (38) can be rewritten as

$$
\mathcal{L}_{\gamma_{S C H C N}}(s)=\sum_{i=1}^{N_{c}} \frac{\alpha_{i}}{\left(s+\lambda_{e q}\right)^{i}}+\frac{\beta_{0}}{s+\lambda_{0}}+\sum_{j=1}^{N-N_{c}} \frac{\beta_{j}}{s+\left(1+\frac{j}{N_{c}}\right) \lambda_{e q}},
$$

where

$$
\begin{gathered}
c=\frac{N !}{N_{c} ! N_{c}^{N-N_{c}}} \lambda_{0} \lambda_{e q}^{N}, \\
\theta_{0}=\frac{1}{\prod_{n=1}^{N-N_{c}}\left[\left(1+\frac{n}{N_{c}}\right) \lambda_{e q}-\lambda_{0}\right]}, \\
\theta_{k}=\frac{1}{\left[-\left(1+\frac{k}{N_{c}}\right) \lambda_{e q}+\lambda_{0}\right] \prod_{n=1, n \neq k}^{N-N_{c}}\left[\left(\frac{n-k}{N_{c}}\right) \lambda_{e q}\right]}, \\
\left.\alpha_{i}=\left.\frac{c}{\left(N_{c}-i\right) !} \frac{d^{N_{c}-i}}{d s^{N_{c}-i}}\left[\frac{1}{\left(s+\lambda_{0}\right) \prod_{n=1}^{N-N_{c}}\left[s+\left(1+\frac{n}{N_{c}}\right) \lambda_{e q}\right]}\right]\right|_{s=-\lambda_{e q}} ^{N-N_{c}} \frac{\theta_{k}(-1)^{N_{c}-i}\left(N_{c}-i\right) !}{\left(\frac{k}{N_{c}} \lambda_{e q}\right)^{N_{c}-i+1}}\right], \\
\frac{c}{\left(N_{c}-i\right) !}\left[\frac{\theta_{0}(-1)^{N_{c}-i}\left(N_{c}-i\right) !}{\left(-\lambda_{e q}+\lambda_{0}\right)^{N_{c}-i+1}+\sum_{k=1}^{N}},\right. \\
\beta_{0}=\frac{c}{\left(\lambda_{e q}-\lambda_{0}\right)^{N_{c}} \prod_{n=1}^{N-N_{c}}\left[\left(1+\frac{n}{N_{c}}\right) \lambda_{e q}-\lambda_{0}\right]},
\end{gathered}
$$

and

$$
\beta_{j}=\frac{c}{\left(-\frac{j}{N_{c}} \lambda_{e q}\right)^{N_{c}}\left[-\left(1+\frac{j}{N_{c}}\right) \lambda_{e q}+\lambda_{0}\right] \prod_{n=1, n \neq j}^{N-N_{c}}\left[\left(\frac{n-j}{N_{c}}\right) \lambda_{e q}\right]} .
$$

Applying the inverse Laplace transforms in Eq. (39), we can obtain the PDF of $\gamma_{S C H C N}$ in closed form as [46, Eq. (17.13.17)]

$$
\begin{aligned}
f_{\gamma_{S C H C N}}(\gamma) & =\mathcal{L}^{-1}\left[\mathcal{L}_{\gamma_{S C H C N}}(s)\right] \\
& =\sum_{i=1}^{N_{c}} \frac{\alpha_{i}}{(i-1) !} \gamma^{i-1} e^{-\lambda_{e q} \gamma}+\beta_{0} e^{-\lambda_{0} \gamma}+\sum_{j=1}^{N-N_{c}} \beta_{j} e^{-\left(1+\frac{j}{N_{c}}\right) \lambda_{e q} \gamma} .
\end{aligned}
$$

The CDF of $\gamma_{S C H C N}$ can be obtained directly by integrating the PDF of $\gamma_{S C H C N}$ in closed form as

$$
\begin{aligned}
F_{\gamma_{S C H C N}}(\gamma)= & \int_{0}^{\gamma} f_{\gamma_{S C H C N}}(t) \mathrm{d} t \\
= & \sum_{i=1}^{N_{c}} \frac{\alpha_{i}}{(i-1) !} g\left(i-1, \lambda_{e q}, \gamma\right)+\beta_{0} g\left(0, \lambda_{0}, \gamma\right)+\sum_{j=1}^{N-N_{c}} \beta_{j} \cdot g\left(0,\left(1+\frac{j}{N_{c}}\right) \lambda_{e q}, \gamma\right) \\
= & \sum_{i=1}^{N_{c}} \frac{\alpha_{i}}{\lambda_{e q}^{i}}\left[1-e^{-\lambda_{e q} \gamma} \sum_{m=0}^{i-1} \frac{\left(\lambda_{e q} \gamma\right)^{m}}{m !}\right]+\sum_{j=1}^{N-N_{c}} \frac{\beta_{j}}{\left(1+\frac{j}{N_{c}}\right) \lambda_{e q}}\left(1-e^{-\left(1+\frac{j}{N_{c}}\right) \lambda_{e q} \gamma}\right) \\
& \quad+\frac{\beta_{0}}{\lambda_{0}}\left(1-e^{-\lambda_{0} \gamma}\right),
\end{aligned}
$$


where $g(n, \beta, \gamma)$ is defined as [46, Eq. (3.351.1)]

$$
\begin{aligned}
g(n, \beta, \gamma)= & \int_{0}^{x} e^{-\beta t} t^{n} \mathrm{~d} t \\
= & \frac{n !}{\beta^{n+1}}\left[1-e^{-\beta x} \sum_{m=0}^{n} \frac{(\beta x)^{m}}{m !}\right], \\
& {[\gamma>0, \operatorname{Re} \beta>0, n=0,1,2, \ldots] . }
\end{aligned}
$$

2) Case of $\lambda_{0}=\lambda_{e q}$ : Eq. (38) can be rewritten as

$$
\begin{aligned}
\mathcal{L}_{\gamma_{S C H C N}}(s) & =\mathcal{L}_{\gamma_{\text {coop }}}(s) \cdot \mathcal{L}_{\gamma_{0}}(s) \\
& =\frac{\frac{N !}{N_{c} ! N_{c}^{N-N_{c}}} \lambda_{0}^{N+1}}{\left(s+\lambda_{0}\right)^{N_{c}+1} \prod_{n=1}^{N-N_{c}}\left[s+\left(1+\frac{n}{N_{c}}\right) \lambda_{0}\right]} .
\end{aligned}
$$

Hence, applying the partial fraction expansion in Eq. (49), we can further simplify it as

$$
\mathcal{L}_{\gamma_{S C H C N}}(s)=\sum_{i=1}^{N_{c}+1} \frac{\alpha_{i}^{*}}{\left(s+\lambda_{0}\right)^{i}}+\sum_{j=1}^{N-N_{c}} \frac{\beta_{j}^{*}}{s+\left(1+\frac{j}{N_{c}}\right) \lambda_{0}},
$$

where

$$
\begin{gathered}
c^{*}=\frac{N !}{N_{c} ! N_{c}^{N-N_{c}}} \lambda_{0}^{N+1}, \\
\theta_{k}^{*}=\frac{1}{\prod_{n=1, n \neq k}^{N-N_{c}}\left[\left(\frac{n-k}{N_{c}}\right) \lambda_{0}\right]}, \\
\alpha_{i}^{*}=\left.\frac{c^{*}}{\left(N_{c}+1-i\right) !} \frac{d^{N_{c}+1-i}}{d s^{N_{c}+1-i}}\left[\frac{1}{\prod_{n=1}^{N-N_{c}}\left[s+\left(1+\frac{n}{N_{c}}\right) \lambda_{0}\right]}\right]\right|_{s=-\lambda_{0}} \\
=\frac{c^{*}}{\left(N_{c}+1-i\right) !}\left[\sum_{k=1}^{N-N_{c}} \frac{\theta_{k}^{*}(-1)^{N_{c}+1-i}\left(N_{c}+1-i\right) !}{\left(\frac{k}{N_{c}} \lambda_{0}\right)^{N_{c}-i+2}}\right]
\end{gathered}
$$

and

$$
\beta_{j}^{*}=\frac{c^{*}}{\left(-\frac{j}{N_{c}} \lambda_{0}\right)^{N_{c}+1} \prod_{n=1, n \neq j}^{N-N_{c}}\left[\left(\frac{n-j}{N_{c}}\right) \lambda_{0}\right]} .
$$

Similarly, we can derive the expression of the PDF and CDF of $\gamma_{S C H C N}$ as follows:

$$
f_{\gamma_{S C H C N}}(\gamma)=\mathcal{L}^{-1}\left[\mathcal{L}_{\gamma_{S C H C N}}(s)\right]=\sum_{i=1}^{N_{c}+1} \frac{\alpha_{i}^{*}}{(i-1) !} \gamma^{i-1} e^{-\lambda_{0} \gamma}+\sum_{j=1}^{N-N_{c}} \beta_{j}^{*} e^{-\left(1+\frac{j}{N_{c}}\right) \lambda_{0} \gamma}
$$

and

$$
\begin{aligned}
F_{\gamma_{S C H C N}}(\gamma) & =\int_{0}^{\gamma} f_{\gamma_{S C H C N}}(t) \mathrm{d} t \\
& =\sum_{i=1}^{N_{c}+1} \frac{\alpha_{i}^{*}}{\lambda_{e q}^{i}}\left[1-e^{-\lambda_{0} \gamma} \sum_{m=0}^{i-1} \frac{\left(\lambda_{0} \gamma\right)^{m}}{m !}\right]+\sum_{j=1}^{N-N_{c}} \frac{\beta_{j}^{*}}{\left(1+\frac{j}{N_{c}}\right) \lambda_{0}}\left(1-e^{-\left(1+\frac{j}{N_{c}}\right) \lambda_{0} \gamma}\right) .
\end{aligned}
$$

Hence, Property 2 is proved. 


\section{APPENDIX D}

\section{PROOF OF PROPERTY 3}

The Laplace transform of the PDF of $\gamma_{S C H C N}$ is derived in Appendix $\mathrm{C}$ as

$$
\mathcal{L}_{\gamma_{S C H C N}}(s)=\frac{\frac{N !}{N_{c} ! N_{c}^{N-N_{c}}} \lambda_{0} \lambda_{e q}^{N}}{\left(s+\lambda_{e q}\right)^{N_{c}}\left(s+\lambda_{0}\right) \prod_{n=1}^{N-N_{c}}\left[s+\left(1+\frac{n}{N_{c}}\right) \lambda_{e q}\right]} .
$$

Since the Laplace transform of the PDF and CDF of $\gamma_{S C H C N}$ are related by

$$
\hat{\mathcal{L}}_{\gamma_{S C H C N}}(s)=\frac{\mathcal{L}_{\gamma_{S C H C N}}(s)}{s},
$$

where $\hat{\mathcal{L}}_{\gamma_{S C H C N}}(s)$ is the Laplace transform of the CDF of $\gamma_{S C H C N}$. Applying the inverse Laplace transforms, the CDF of $\gamma_{S C H C N}$ can be expressed as

$$
\begin{aligned}
F_{\gamma_{S C H C N}}(\gamma) & =\mathcal{L}^{-1}\left[\frac{\mathcal{L}_{\gamma_{S C H C N}}(s)}{s}\right] \\
& =\mathcal{L}^{-1}\left[\frac{\frac{N !}{N_{c} ! N_{c}^{N-N_{c}}} \lambda_{0} \lambda_{e q}^{N}}{s\left(s+\lambda_{e q}\right)^{N_{c}}\left(s+\lambda_{0}\right) \prod_{n=1}^{N-N_{c}}\left[s+\left(1+\frac{n}{N_{c}}\right) \lambda_{e q}\right]}\right] .
\end{aligned}
$$

At high SNR, $\bar{\gamma}_{0}$ and $\bar{\gamma}_{e q}$ are large. According to $\lambda_{0}=1 / \bar{\gamma}_{0}$ and $\lambda_{e q}=1 / \bar{\gamma}_{e q}$, we know that $\lambda_{0}$ and $\lambda_{e q}$ are small. Hence, Eq. (59) can be simplified as [46, Eq. (17.13.2)]

$$
\begin{aligned}
F_{\gamma_{S C H C N}}(\gamma) & \approx \mathcal{L}^{-1}\left[\frac{N !}{N_{c} ! N_{c}{ }^{N-N_{c}}} \frac{\lambda_{0} \lambda_{e q}{ }^{N}}{s^{N+2}}\right] \\
& =\frac{1}{(N+1) N_{c} ! N_{c}{ }^{N-N_{c}}} \lambda_{0} \lambda_{e q}{ }^{N} \gamma^{N+1} .
\end{aligned}
$$

Hence, Eq. (29) is proved.

\section{REFERENCES}

[1] Z. Wang, Y. Chen, and C. Li, "CORMAN: A novel cooperative opportunistic routing scheme in mobile ad hoc networks," IEEE J. Sel. Areas Commun., vol. 30, no. 2, pp. 289-296, Feb. 2012.

[2] X. Cheng, C.-X. Wang, H. Wang, X. Gao, X.-H. You, D. Yuan, B. Ai, Q. Huo, L.-Y. Song, and B.-L. Jiao, "Cooperative MIMO channel modeling and multi-link spatial correlation properties," IEEE J. Sel. Areas Commun., vol. 30, no. 2, pp. 388-396, Feb. 2012.

[3] A. Sendonaris, E. Erkip, and B. Aazhang, "User cooperation diversity. Part I. System description," IEEE Trans. Commun., vol. 51, no. 11, pp. 1927-1938, Nov. 2003.

[4] —_, "User cooperation diversity. Part II. Implementation aspects and performance analysis," IEEE Trans. Commun., vol. 51, no. 11, pp. 1939-1948, Nov. 2003.

[5] J. Laneman, D. Tse, and G. Wornell, "Cooperative diversity in wireless networks: Efficient protocols and outage behavior," IEEE Trans. Inf. Theory, vol. 50, no. 12, pp. 3062-3080, Dec. 2004.

[6] F. Onat, A. Adinoyi, Y. Fan, H. Yanikomeroglu, J. Thompson, and I. Marsland, "Threshold selection for SNR-based selective digital relaying in cooperative wireless networks," IEEE Trans. Wireless Commun., vol. 7, no. 11, pp. 4226-4237, Nov. 2008. 
[7] T. Liu, L. Song, and B. Jiao, "Optimum power allocation for hybrid relay selection schemes," in Proc. IEEE Int. Conf. Wireless Commun. and Signal Process. (WCSP'09), Nov. 2009, pp. 1 -5.

[8] T. Duong and H.-J. Zepernick, "Hybrid decode-amplify-forward cooperative communications with multiple relays," in Proc. IEEE Wireless Commun. and Networking Conf. (WCNC'09), Apr. 2009, pp. 1-6.

[9] T. Duong and H. Zepernick, "On the performance gain of hybrid decode-amplify-forward cooperative communications," EURASIP J. Wireless Commun. and Networking, vol. 2009, pp. 1-10, 2009.

[10] F. Onat, A. Adinoyi, Y. Fan, H. Yanikomeroglu, and J. Thompson, "Optimum threshold for SNR-based selective digital relaying schemes in cooperative wireless networks," in Proc. IEEE Wireless Commun. and Networking Conf. (WCNC'07), Mar. 2007, pp. 969-974.

[11] F. Onat, Y. Fan, H. Yanikomeroglu, and H. Poor, "Threshold based relay selection in cooperative wireless networks," in Proc. IEEE Global Telecommun. Conf. (Globecom'08), Nov./Dec. 2008, pp. 1-5.

[12] F. Onat, Y. Fan, H. Yanikomeroglu, and J. Thompson, "Asymptotic BER analysis of threshold digital relaying schemes in cooperative wireless systems," IEEE Trans. Wireless Commun., vol. 7, no. 12, pp. 4938-4947, Dec. 2008.

[13] T. Wang, A. Cano, and G. B. Giannakis, "Efficient demodulation in cooperative schemes using decode-and-forward relays," in Proc. 39th Asilomar Conf. on Signals, Syst. and Comput., Oct. 2005, pp. 1051-1055.

[14] T. Wang, A. Cano, G. Giannakis, and J. Laneman, "High-performance cooperative demodulation with decode-and-forward relays," IEEE Trans. Commun., vol. 55, no. 7, pp. 1427-1438, Jul. 2007.

[15] T. Wang, G. Giannakis, and R. Wang, "Smart regenerative relays for link-adaptive cooperative communications," IEEE Trans. Commun., vol. 56, no. 11, pp. 1950-1960, Nov. 2008.

[16] N. Vien, H. Nguyen, and T. Le-Ngoc, "Diversity analysis of smart relaying," IEEE Trans. Veh. Technol., vol. 58, no. 6, pp. 2849-2862, Jul. 2009.

[17] T. Kwon, S. Lim, W. Seo, and D. Hong, "LLR-based symbol selective transmission with a near-optimal threshold to minimize BEP for demodulation-forward relay systems," IEEE Trans. Wireless Commun., vol. 9, no. 2, pp. 540-545, Feb. 2010.

[18] X. Bao and J. Li, "Decode-amplify-forward (DAF): a new class of forwarding strategy for wireless relay channels," in Proc. IEEE Workshop Signal Process. Advances in Wireless Commun., Jun. 5-8, 2005, pp. 816-820.

[19] — - "Efficient message relaying for wireless user cooperation: Decode-amplify-forward (DAF) and hybrid DAF and coded-cooperation,” IEEE Trans. Wireless Commun., vol. 6, no. 11, pp. 3975-3984, Nov. 2007.

[20] B. Can, H. Yomo, and E. De Carvalho, "Hybrid forwarding scheme for cooperative relaying in OFDM based networks," in Proc. IEEE Int. Conf. Commun. (ICC'06), Jun. 2006, pp. 4520-4525.

[21] Y. Li, B. Vucetic, Z. Chen, and J. Yuan, “An improved relay selection scheme with hybrid relaying protocols," in Proc. IEEE Global Telecommun. Conf. (Globecom'07), Nov. 2007, pp. 3704-3708.

[22] L. Song, Y. Li, M. Tao, and A. Vasilakos, "A hybrid relay selection scheme using differential modulation," in Proc. IEEE Wireless Commun. and Networking Conf. (WCNC'09), Apr. 2009, pp. 1-6.

[23] T. Liu, L. Song, B. Jiao, and Y. Zhang, "Symbol error rate analysis and power allocation for adaptive relay selection schemes," Wireless Personal Commun., vol. 56, pp. 457-467, 2011.

[24] J. Laneman and G. Wornell, "Distributed space-time-coded protocols for exploiting cooperative diversity in wireless networks," IEEE Trans. Inf. Theory, vol. 49, no. 10, pp. 2415-2425, Oct. 2003.

[25] P. Anghel and M. Kaveh, "Exact symbol error probability of a cooperative network in a rayleigh-fading environment," IEEE Trans. Wireless Commun., vol. 3, no. 5, pp. 1416-1421, Sep. 2004. 
[26] A. Ribeiro, X. Cai, and G. Giannakis, "Symbol error probabilities for general cooperative links," IEEE Trans. Wireless Commun., vol. 4, no. 3, pp. 1264-1273, May 2005.

[27] Y. Zhao, R. Adve, and T. J. Lim, "Symbol error rate of selection amplify-and-forward relay systems," IEEE Commun. Lett., vol. 10, no. 11, pp. 757-759, Nov. 2006.

[28] Y. Zhao, R. Adve, and T. Lim, "Improving amplify-and-forward relay networks: optimal power allocation versus selection," IEEE Trans. Wireless Commun., vol. 6, no. 8, pp. 3114-3123, Aug. 2007.

[29] A. Sulyman and M. Kousa, "Bit error rate performance of a generalized diversity selection combining scheme in Nakagami fading channels," in Proc. IEEE Wireless Commun. and Networking Conf. (WCNC' 2000), 2000, pp. 1080 -1085.

[30] Y. Chen and C. Tellambura, "A new hybrid generalized selection combining scheme and its performance over fading channels," in Proc. IEEE Wireless Commun. and Networking Conf. (WCNC'04), Mar. 2004, pp. 926 - 931 Vol.2.

[31] W. Li and N. Beaulieu, "A study of generalized receiver selection combining schemes for Alamouti MIMO systems with MPSK," in Proc. IEEE Int. Conf. Commun. (ICC'06), Jun. 2006, pp. $4230-4235$.

[32] N. Kong, T. Eng, and L. Milstein, “A selection combining scheme for RAKE receivers," in Proc. IEEE Int. Conf. Universal Personal Commun., Nov. 1995, pp. $426-430$.

[33] S. Ikki and M. Ahmed, "Performance analysis of generalized selection combining for amplify-and-forward cooperativediversity networks," in Proc. IEEE Int. Conf. Commun. (ICC'09), Jun. 2009, pp. 1-6.

[34] — - "Performance analysis of generalized selection combining for decode-and-forward cooperative-diversity networks," in Proc. IEEE Veh. Technol. Conf. Fall (VTC'10-Fall), Sep. 2010, pp. 1-5.

[35] E. Beres and R. Adve, "On selection cooperation in distributed networks," in Proc. 40th Annu. Conf. Inf. Sci. and Syst., Mar. 22-24, 2006, pp. 1056-1061.

[36] Z. Yi and I. Kim, "Diversity order analysis of the decode-and-forward cooperative networks with relay selection," IEEE Trans. Wireless Commun., vol. 7, no. 5, pp. 1792-1799, May 2008.

[37] D. Soldani and S. Dixit, "Wireless relays for broadband access [radio communications series]," IEEE Commun. Mag., vol. 46, no. 3, pp. 58-66, Mar. 2008.

[38] T. Liu, L. Song, Y. Li, Q. Huo, and B. Jiao, "Performance analysis of hybrid relay selection in cooperative wireless systems," IEEE Trans. Commun., vol. 60, no. 3, pp. 779-788, Mar. 2012.

[39] T. S. Rappaport, Wireless communications: principles and practice, 2nd ed. Upper Saddle River, NJ: Prentice Hall PTR, 2002.

[40] J. Proakis, Digital Communications. New York: McGraw-hill, 2001.

[41] H. El Gamal and J. Hammons, A.R., "Analyzing the turbo decoder using the Gaussian approximation," IEEE Trans. Inf. Theory, vol. 47, no. 2, pp. 671-686, Feb. 2001.

[42] I. Chatzigeorgiou, I. Wassell, and R. Carrasco, "On the frame error rate of transmission schemes on quasi-static fading channels," in Proc. 42nd Annu. Conf. Inf. Sciences and Syst. (CISS '08), Mar. 2008, pp. 577 -581.

[43] — , "Threshold-based frame error rate analysis of MIMO systems over quasistatic fading channels," Electronics Lett., vol. 45, no. 4, pp. 216-217, Dec. 2009.

[44] L. Zheng and D. Tse, "Diversity and multiplexing: a fundamental tradeoff in multiple-antenna channels," IEEE Trans. Inf. Theory, vol. 49, no. 5, pp. 1073-1096, May. 2003.

[45] M. Rodrigues, I. Chatzigeorgiou, I. Wassell, and R. Carrasco, "Performance analysis of turbo codes in quasi-static fading channels," IET Commun., vol. 2, no. 3, pp. 449-461, Mar. 2008.

[46] I. Gradshteyn, I. Ryzhik, A. Jeffrey, and D. Zwillinger, Table of Integrals, Series and Products. Academic press, 2007. 
[47] S. Alamouti, "A simple transmit diversity technique for wireless communications," IEEE J. Sel. Areas Commun., vol. 16, no. 8, pp. 1451-1458, Oct. 1998.

[48] P. Lettieri and M. Srivastava, "Adaptive frame length control for improving wireless link throughput, range, and energy efficiency," in Proc. IEEE INFOCOM'98, Mar. 1998, pp. 564-571.

[49] H. David and H. Nagaraja, Order statistics. Wiley-Interscience, 2003.

[50] M. Simon and M. Alouini, Digital Communication over Fading Channels. Wiley-IEEE Press, 2005.

[51] B. Latni, Signal processing and linear systems. USA: Oxford University Press, 1998.

[52] Q. Huo, T. Liu, L. Song, and B. Jiao, "All-participate hybrid forward cooperative communications with multiple relays," in Proc. IEEE Int. Conf. Wireless Commun. and Signal Process. (WCSP'10), Suzhou, P.R. China, Oct. 2010, pp. 1-6.

[53] Q. Huo, L. Song, Y. Li, and B. Jiao, "A distributed differential space-time coding scheme with analog network coding in two-way relay networks," IEEE Trans. Signal Process., vol. 60, no. 9, pp. 4998-5004, Sep. 2012.

[54] _ _ "Novel multihop transmission schemes using selective network coding and differential modulation for two-way relay networks," in Proc. IEEE Int. Conf. Commun. (ICC’13), Budapest, Hungary, Jun. 2013, pp. 5924-5928.

[55] Q. Huo, T. Liu, L. Song, and B. Jiao, "Hybrid forward scheme with generalized selective combining," in Proc. IEEE Int. Conf. Wireless Commun. and Signal Process. (WCSP'11), Nanjing, P.R. China, Nov. 2011, pp. 1-5. 
TABLE I

SNR THRESHOLD

\begin{tabular}{|c|c|c|c|}
\hline Diversity Order: $d$ & Frame Length: $L$ & Proposed SNR Threshold: $\gamma_{t, d}, \mathrm{~dB}$ & SNR Threshold of [42]: $\gamma_{t}, \mathrm{~dB}$ \\
\hline \hline 1 & 100 & 5.10 & \multirow{2}{*}{4} \\
\cline { 1 - 3 } 2 & 100 & 5.36 & \multirow{2}{*}{} \\
\cline { 1 - 3 } & 100 & 5.62 & \\
\hline 4 & 100 & 5.89 & 5.50 \\
\hline 4 & 200 & 6.45 & 6.24 \\
\hline 4 & 400 & 6.97 & \multirow{2}{*}{6} \\
\hline
\end{tabular}

TABLE II

SCENARIOS

\begin{tabular}{|c|c|c|c|c|}
\hline & System Type & Number of Nodes & Diversity Order: $d$ & SNRs \\
\hline \hline case 0 & MIMO & $N_{T}=1, N=N_{R}=1,2,4$ & $d=1,2,4$ & $\Omega=1$ \\
\hline case 1 & SCHCN & $N=1,2,3$ & $d=2,3,4$ & $\Omega_{0}=\Omega_{1 i}=\Omega_{2 i}=1$ \\
\hline case 2 & SCHCN & $N=3$ & $d=4$ & $\Omega_{0}=1, \Omega_{1 i}=16, \Omega_{2 i}=1$ \\
\hline case 3 & SCHCN & $N=3$ & $d=4$ & $\Omega_{0}=1, \Omega_{1 i}=1 / 16, \Omega_{2 i}=1$ \\
\hline
\end{tabular}

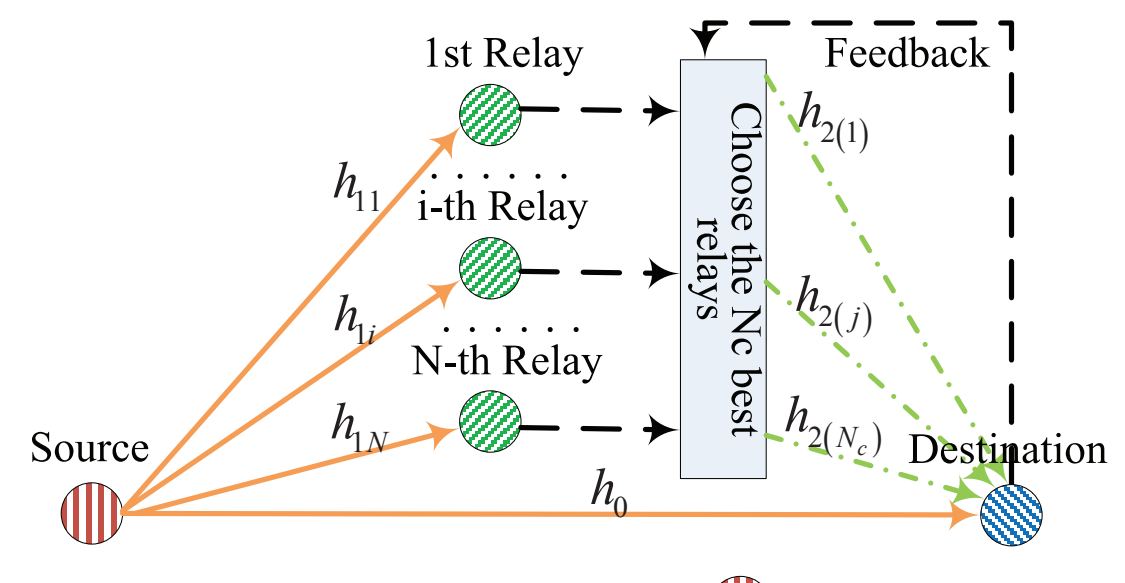

$\longrightarrow$ 1st phase transmission

Source

$-\cdot \rightarrow$ 2nd phase transmission

Relay

$\longrightarrow$ Feedback \& relay selection

Destination

Fig. 1. Block diagram of the SCHCN scheme. 


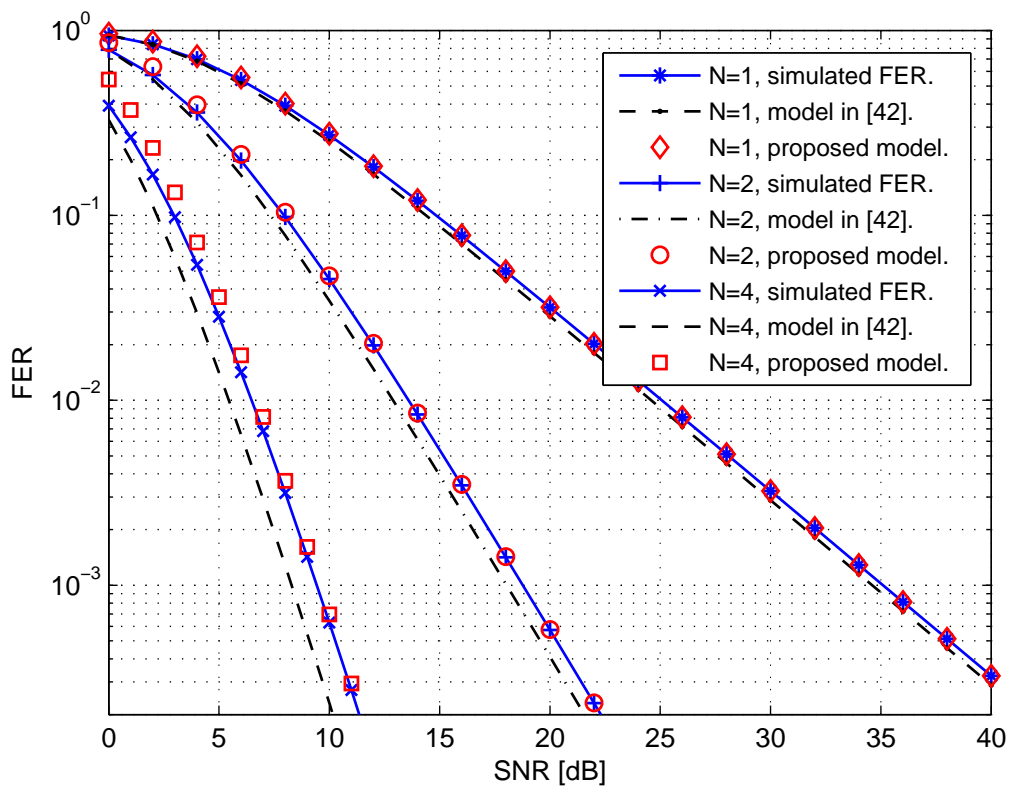

Fig. 2. FER comparison of the proposed model and the model of [42] for case 0: general MIMO channels with $N_{T}=1$, $N=N_{R}=1,2,4, \Omega=1$ and $L=100$.

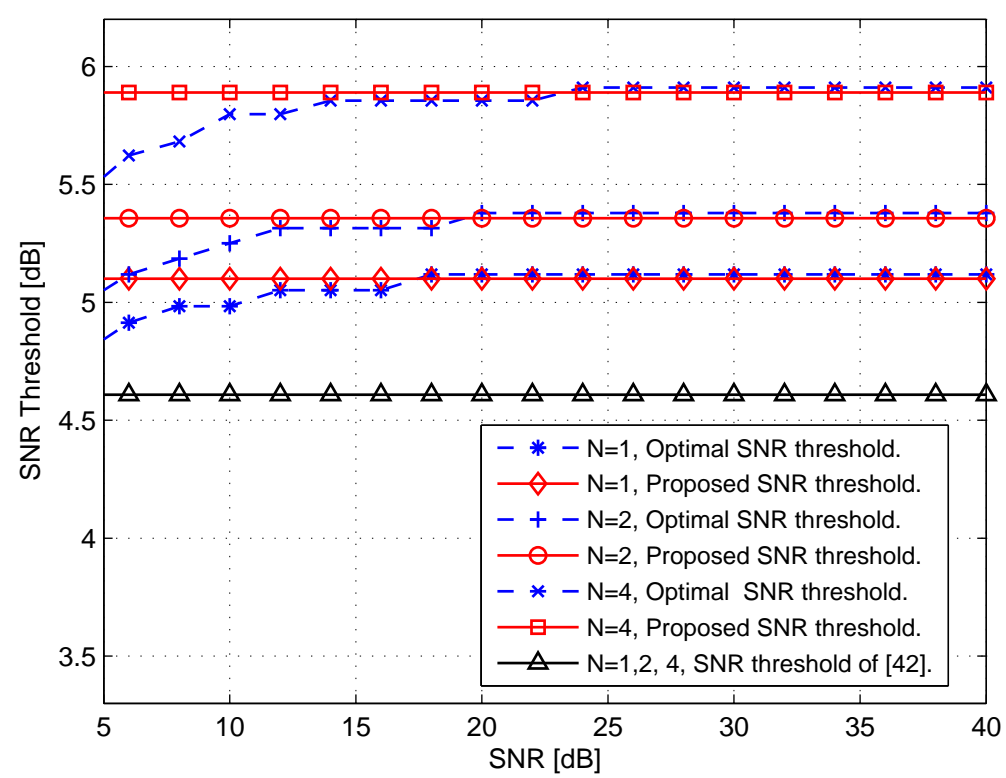

Fig. 3. SNR threshold comparison of the proposed model and the model of [42] for case 0: general MIMO channels with $N_{T}=1, N=N_{R}=1,2,4, \Omega=1$ and $L=100$. 


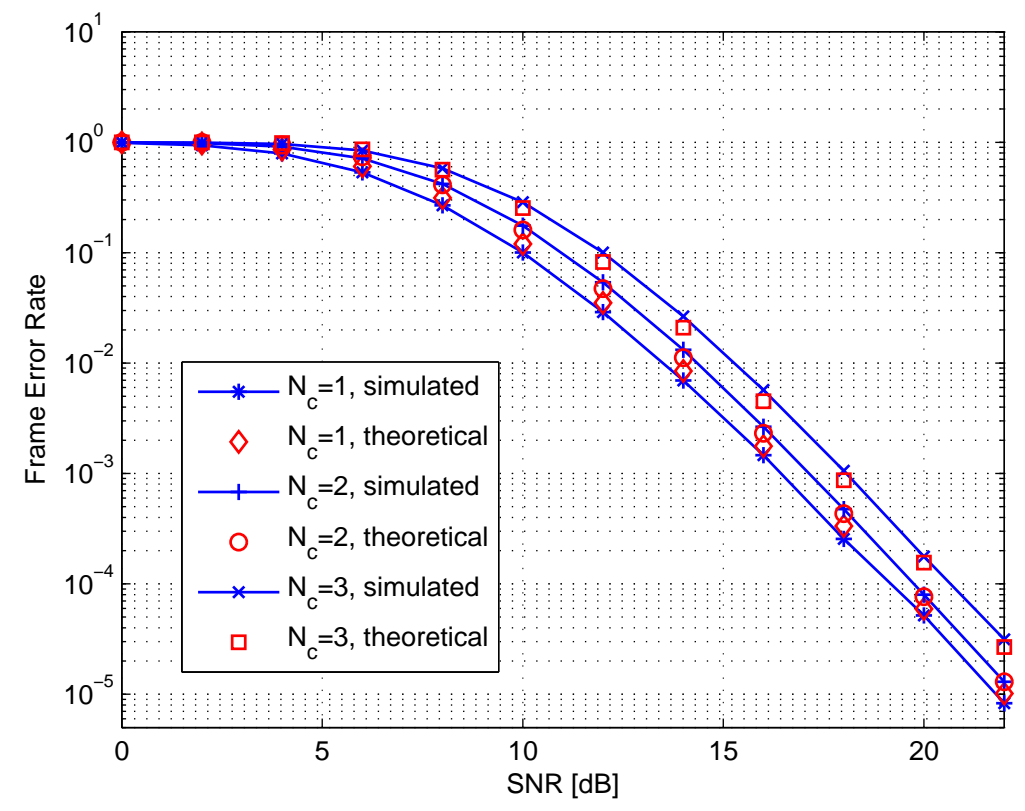

Fig. 4. Closed-form and simulated FER results of the SCHCN scheme under a total transmit power constraint: $N=3$, $\Omega_{0}=\Omega_{1 i}=\Omega_{2 i}=1$ and $L=100$.

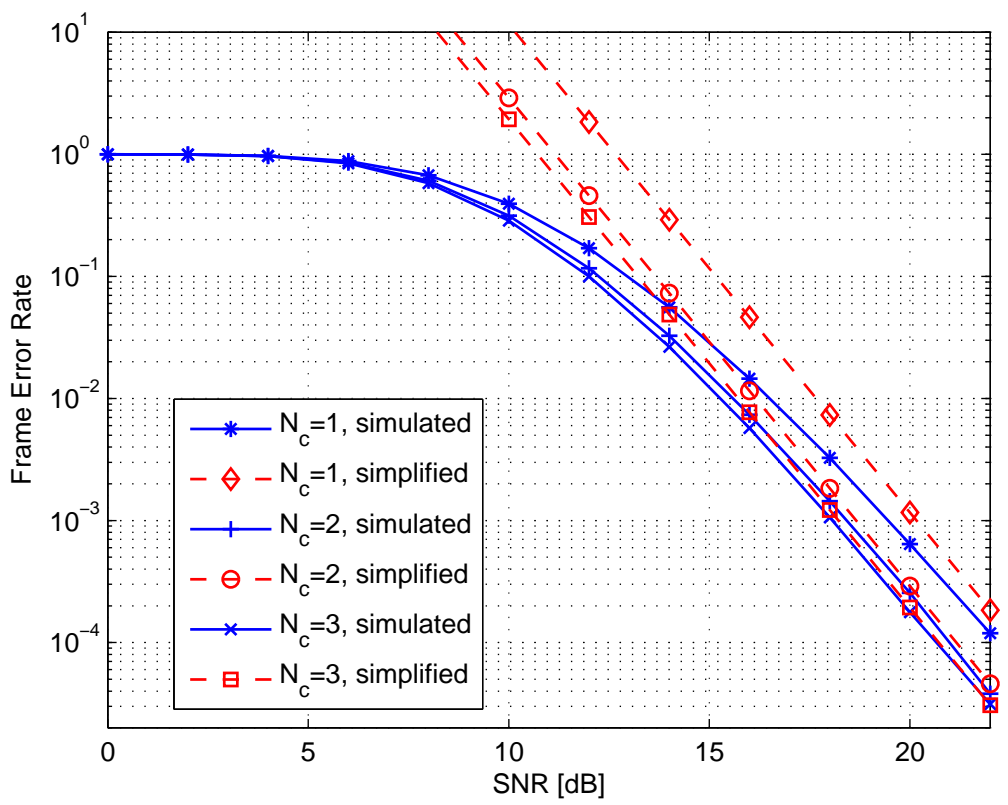

Fig. 5. Asymptotic and simulated FER results of the SCHCN scheme under an individual power constraint: $N=3, \Omega_{0}=$ $\Omega_{1 i}=\Omega_{2 i}=1$ and $L=100$. 


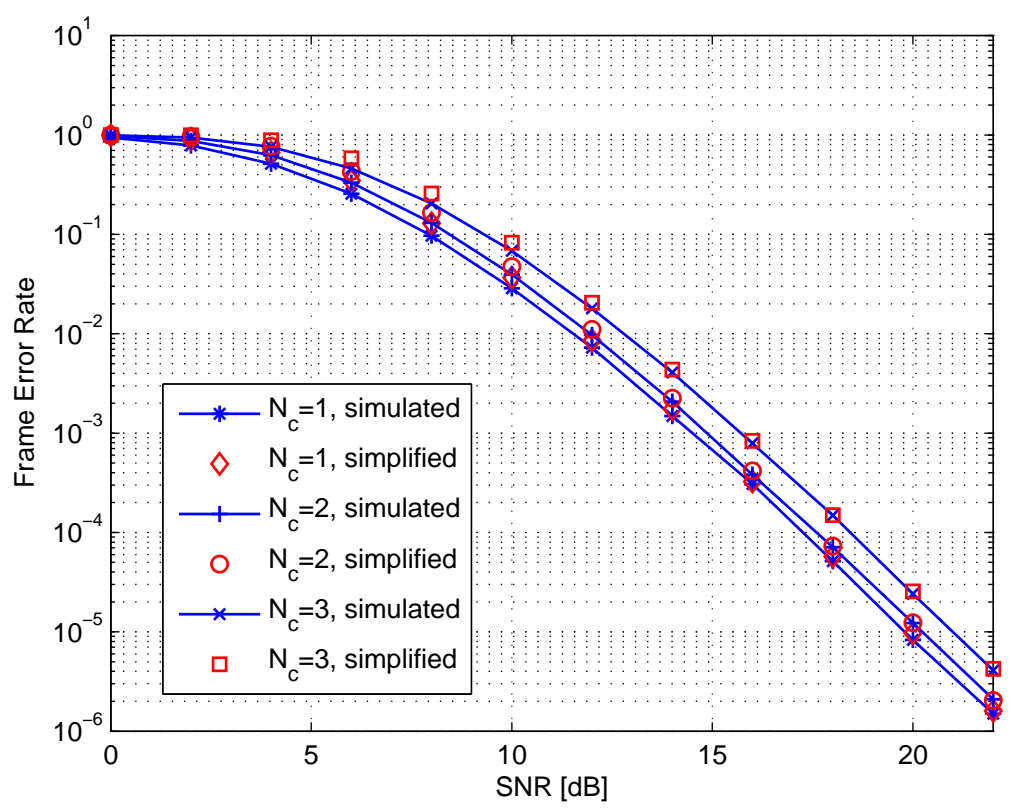

Fig. 6. Closed-form and simulated FER results of the SCHCN scheme under a total transmit power constraint: $N=3, \Omega_{0}=1$, $\Omega_{1 i}=16, \Omega_{2 i}=1$ and $L=100$.

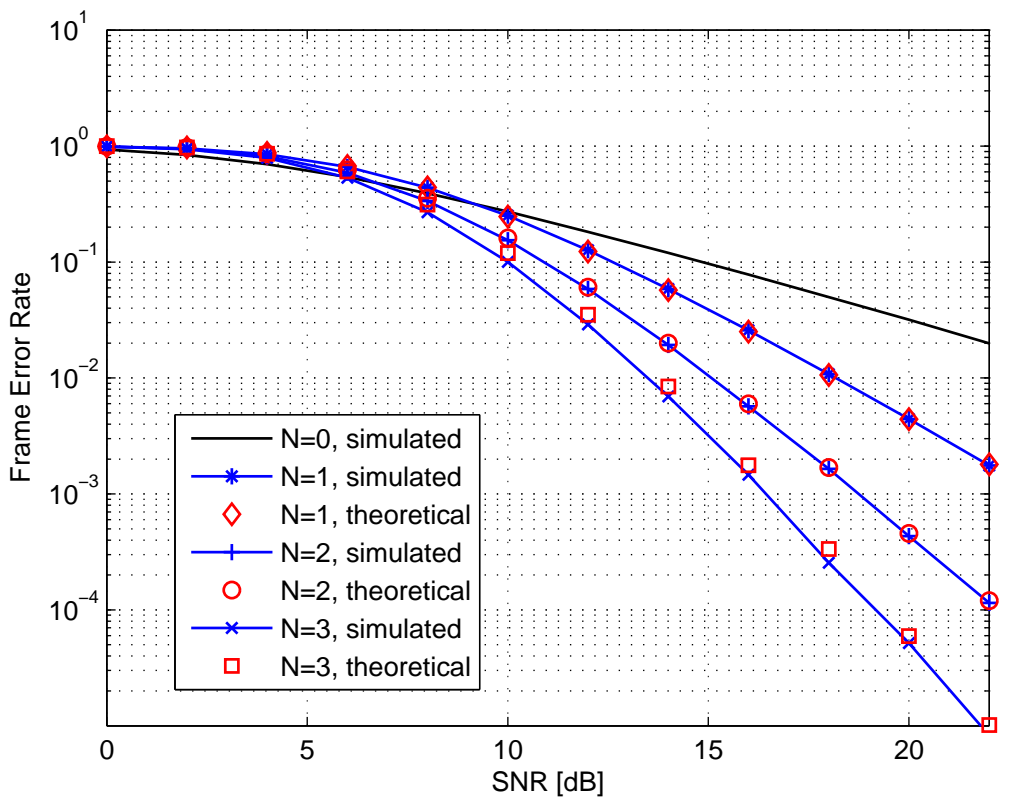

Fig. 7. Closed-form and simulated FER results of the SCHCN scheme under a total transmit power constraint $(N=1,2,3$, $\left.N_{c}=1\right)$ and non-cooperative system $(N=0), \Omega_{0}=\Omega_{1 i}=\Omega_{2 i}=1$ and $L=100$. 


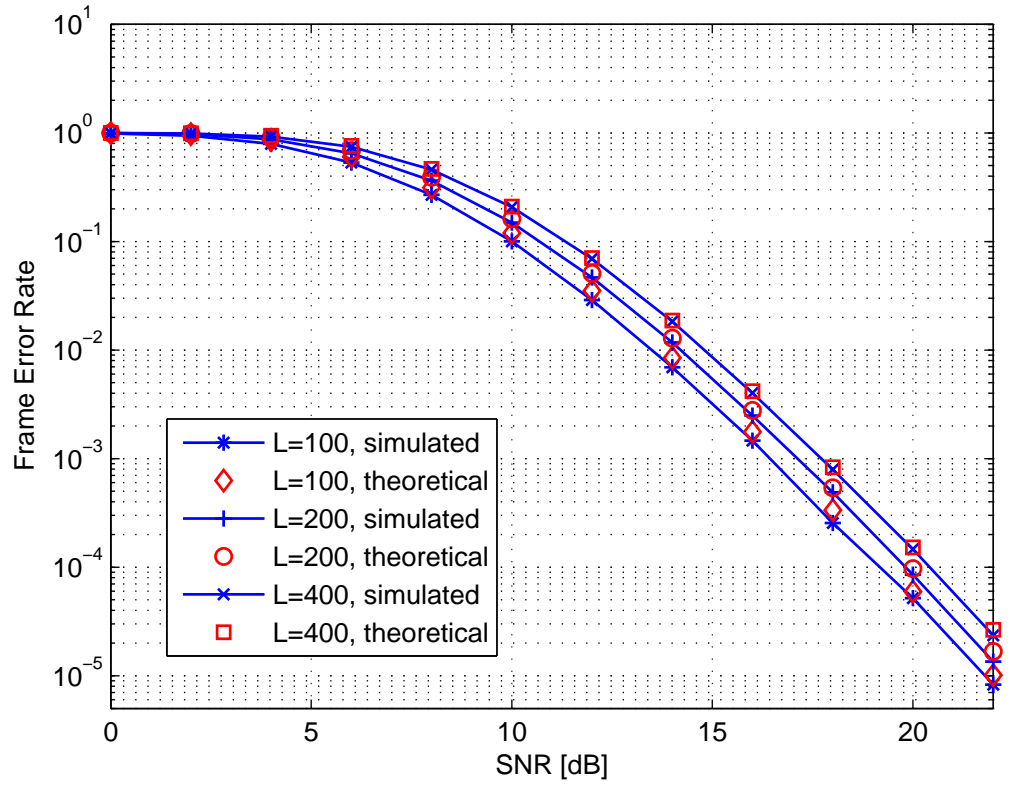

Fig. 8. Closed-form and simulated FER results of the SCHCN scheme under a total transmit power constraint: $N=3, N_{c}=1$, $\Omega_{0}=\Omega_{1 i}=\Omega_{2 i}=1$ and $L=100,200,400$. 